statement is obviously true; if $U_{1}$ lies in $\left[U_{1}\right], A=U_{1} U_{1}^{*} P_{1} U_{1}$ and, since $U_{1}^{*} P_{1} U_{1}$ is a positive hermitian matrix of rank $r$, $U_{1}^{*} P_{1} U_{1}=P_{2}$ and $U_{1}$ lies in $\left[U_{2}\right]$. Similarly any member $U_{2}$ of $\left[U_{2}\right]$ lies in $\left[U_{1}\right]$. Further the matrix $P_{2}$ is invariant under unitary transformation by any matrix of the group $G_{1}$, and $P_{1}$ under transformation by any matrix of the group $G_{2}$. For if $Z_{1}$ lies in $G_{1}, A Z_{1}=A$ so that $A=U_{2} Z_{1} Z_{1}{ }^{*} P_{2} Z_{1}$, and accordingly, $Z_{1}^{*} P_{2} Z_{1}=P_{2}$.

The Johns Hopkins University

\title{
ON A THEOREM OF FERAUD
}

BY D. C. LEWIS, JR.*

The Birkhoff-Pfaffian equations of dynamics are written in variational form as follows:

$$
\delta \int\left[\sum_{i=1}^{2 m} X_{i}\left(\frac{d x_{i}}{d t}\right)+Q\right] d t=0,
$$

where $Q$ and the $X$ 's are functions of $x_{1}, \cdots, x_{2 m}$ and, in general, depend also periodically upon $t$, and where the skew-symmetric determinant $\left|a_{i j}\right|,\left(a_{i j}=\partial X_{i} / \partial x_{j}-\partial X_{j} / \partial x_{i}\right)$, does not vanish in the regions considered. We restrict attention to the neighborhood of a generalized equilibrium point, that is, a point where all the $\partial Q / \partial x_{i}-\partial X_{i} / \partial t$ vanish identically in $t$. We take this point at the origin, $x_{i}=0,(i=1,2, \cdots, 2 m)$.

The problem of reducing the Pfaffian system to a Hamiltonian system can be reduced to that of finding a non-singular transformation, $x_{i}=x_{i}\left(y_{1}, \cdots, y_{2}\right)$, leaving the origin invariant (and depending in general periodically upon $t$ ) which reduces the linear differential form $\sum_{i=1}^{2 m} X_{i} d x_{i}$ to the form $\sum_{i=1}^{m} y_{2 i} d y_{2 i-1}$ $+d w$, where $d w$ is an exact differential in $y_{1}, \cdots, y_{2}$, the coefficients of which are independent of $t$. This same problem also will play an important role in a future paper of mine on "conservative" transformations in $2 m$-dimensional spaces.

The problem has been considered by Féraud, $\uparrow$ who obtained a

* National Research Fellow.

$\dagger$ Extension au cas d'un nombre quelconque de degrés de liberté d'une propriété relative aux systèmes Pfaffiens, Comptes Rendus, vol. 190 (1930), pp. 358-360. 
solution based on the fundamental existence theorem of Riquier for systems of partial differential equations. It appears, however, highly desirable to give a more elementary proof based on existence theorems for ordinary differential equations. The result is thus not limited to the analytic case, as it was by the use of Riquier's theorem in Féraud's proof.

Our proof follows a well known line of thought expounded by Goursat.* There are several reasons, however, why Goursat's writings on the reduction of linear differential forms are not immediately applicable. For one thing, the transformations used by him do not necessarily leave the origin invariant. Also there are important cases where his reasoning might fail. $\dagger$ The present treatment does not presuppose a knowledge of Goursat's work.

We split up our proof into a number of easy lemmas.

Lemma 1. Consider a linear differential form $\sum_{i=1}^{n} X_{i} d x_{i}$ which under a non-singular transformation of coordinates, $x_{i}=g_{i}\left(y_{1}, \cdots, y_{n}\right)$, appears also in the form $\sum_{i=1}^{n} Y_{i} d y_{i}$. Then we have the following elementary relations:

$$
\begin{gathered}
Y_{i}=\sum_{j=1}^{n} X_{j}\left(\frac{\partial g_{j}}{\partial y_{i}}\right), \\
\frac{\partial Y_{i}}{\partial y_{r}}-\frac{\partial Y_{r}}{\partial y_{i}}=\sum_{l, j=1}^{n}\left(\frac{\partial X_{j}}{\partial x_{l}}-\frac{\partial X_{l}}{\partial x_{j}}\right) \frac{\partial g_{l}}{\partial y_{r}} \frac{\partial g_{j}}{\partial y_{i}} .
\end{gathered}
$$

The proof of (1) is trivial, while (2) is deduced at once from (1) by differentiation with respect to $y_{r}$, interchange of indices, and subtraction. In the sequel, we shall uniformly set $a_{i j}=\partial X_{i} / \partial x_{j}-\partial X_{j} / \partial x_{i}$, and $b_{i j}=\partial Y_{i} / \partial y_{j}-\partial Y_{j} / \partial y_{i} ;$ so that (2) may also be written in the form

$$
b_{i j}=\sum_{k, l=1}^{n} a_{k l} \frac{\partial g_{k}}{\partial y_{i}} \frac{\partial g_{l}}{\partial y_{j}} .
$$

* Leçons sur le Problème de Pfaff, 1922, especially Chapter 1.

$\dagger$ For example, if the first method of Chapter I is used, care must be taken, in the series of necessary reductions, that at each step the $\lambda_{i}$ (pages 11 and 12) do not all vanish at the origin. The present paper does, in effect, just this. If the more advanced method of Chapter 4 is used, it would be necessary to compute the derived forms. Also the most available results of Chapter 4 are stated only for the analytic case. 
LEMma 2. Consider the system of ordinary differential equations

$$
\frac{d x_{i}}{d y_{n}}=f_{i}\left(x_{1}, \cdots, x_{n}\right), \quad(i=1,2, \cdots, n),
$$

where the $f_{i}$ are defined and of class $C^{\nu}$ in the neighborhood of the origin. Let $g_{i}\left(y_{1}, \cdots, y_{n-1}, y_{n}\right)$ be the solution which satisfies the initial conditions $x_{1}=y_{1}, \cdots, x_{n-1}=y_{n-1}, x_{n}=0$ for $y_{n}=0$. Then the $g$ 's are of class $C^{\nu}$ in the neighborhood of the origin, and, if the equations $x_{i}=g_{i}\left(y_{1}, \cdots, y_{n-1}, y_{n}\right)$ are thought of as defining $a$ transformation $T$ of the neighborhood of the origin into itself, then $T$ is non-singular if $f_{n}(0, \cdots, 0) \neq 0$.

The fact that the $g^{\prime}$ 's are of class $C^{\nu}$ is an immediate consequence of a well known theorem.* The rest of the lemma follows from the obvious relations

$$
\begin{array}{lr}
\left.\frac{\partial g_{i}}{\partial y_{j}}\right|_{0}=\delta_{i j}, & (i=1,2, \cdots, n), \\
\left.\frac{\partial g_{i}}{\partial y_{j}}\right|_{0}=f_{i}(0, \cdots, 0), & (j=1,2, \cdots, n-1) .
\end{array}
$$

For the jacobian of $T$, evaluated at the origin, turns out to be exactly $f_{n}(0, \cdots, 0)$.

Lemma 3. Consider a Pfaffian $\omega=\sum_{i=1}^{2 k} X_{i} d x_{i}$ satisfying the following conditions:

(A) The $X^{\prime}$ 's are of class $C^{\mu}$ in the neighborhood of the origin.

(B) None of the X's vanish at the origin.

(C) The skew-symmetric determinant $\left|a_{i j}\right|$ does not vanish at the origin.

Then there exists a non-singular transformation $x_{i}=g_{i}\left(y_{1}, \cdots, y_{2 k}\right)$ of the neighborhood of the origin into itself such that $\omega$ appears expressed in terms of the $y^{\prime}$ in the form $e^{y_{2 k}}\left[\sum_{i=1}^{2 k-1} A_{i} d y_{i}\right]$, where the $A$ 's are functions of $y_{1}, \cdots, y_{2 k-1}$ satisfying the following conditions:

( $\alpha$ ) The $A_{i}$ are of class $C^{\mu-2}$.

( $\beta)$ None of the $A_{i}$ vanish at the origin.

* See G. A. Bliss, The solutions of differential equations of the first order as functions of their initial values, Annals of Mathematics, vol. 6 (1904-1905), pp. 49-68, especially p. 67. 
$(\gamma)$ The skew-symmetric matrix $\left(c_{i j}\right)$, where $c_{i j}=\partial A_{i} / \partial y_{j}$ $-\partial A_{j} / \partial y_{i}$, is such that there exists a system of functions $\xi_{i}\left(y_{1}, \cdots, y_{2 k-1}\right)$ which satisfy the linear identities $\sum_{j=1}^{2 k-1} c_{i j} \xi_{j} \equiv 0$ and $\sum_{j-1}^{2 k-1} A_{j} \xi_{j} \equiv 1$.

Proof. Consider the equations

$$
\sum_{j=1}^{2 k} a_{i j}\left(\frac{d x_{j}}{d y_{2 k}}\right)=X_{i}, \quad(i=1,2, \cdots, 2 k) .
$$

Since $\left|a_{i j}\right| \neq 0$, we may solve for the derivatives, writing the result in the form $d x_{i} / d y_{2 k}=f_{i}\left(x_{1}, \cdots, x_{2 k}\right)$, where the $f$ 's are of class $C^{\mu-1}$. On account of (5) and (B) it is seen that not all the $f$ 's vanish at the origin. It is no loss of generality to assume $f_{2 k}(0, \cdots, 0) \neq 0$, since a preliminary linear transformation can always be applied, the effect of which is merely to interchange indices in the desired manner. We now apply Lemma 2 to the present system, taking $n=2 k$ and $\nu=\mu-1$. We proceed to show that the functions $g_{i}$, as there defined, satisfy the conditions of the present lemma.

In equations (5) replace $d x_{j} / d y_{2 k}$ by $\partial g_{j} / \partial y_{2 k}$. Multiply each equation by $\partial g_{i} / \partial y_{2 k}$ and sum with respect to $i$. Since $a_{i j}+a_{j i}=0$, the result is $\sum X_{i}\left(\partial g_{i} / \partial y_{2 k}\right)=0$. Hence from (1) we see that

$$
Y_{2 k} \equiv 0 \text {. }
$$

We also have, of course, $\partial Y_{2 k} / \partial y_{i} \equiv 0$, so that (2) yields

$$
\frac{\partial Y_{i}}{\partial y_{2 k}}=\sum_{l, j=1} a_{j l} \frac{\partial g_{l}}{\partial y_{2 k}} \frac{\partial g_{j}}{\partial y_{i}}
$$

and, since the $g$ 's satisfy (5), the right member is equal to $\sum X_{j}\left(\partial g_{j} / \partial y_{i}\right)$, which by (1) is precisely $Y_{i}$. Hence we have the result that $\partial Y_{i} / \partial y_{2 k} \equiv Y_{i}$, or

$$
Y_{i}=e^{y_{2 k}} A_{i},
$$

where $A_{i}$ is a suitably chosen function of $y_{1}, \cdots, y_{2 k-1}$, but independent of $y_{2 k}$. From (6) and (7) we have

$$
\omega=\sum_{i=1}^{2 k} Y_{i} d y_{i}=e^{y_{2 k}}\left[\sum_{i=1}^{2 k-1} A_{i} d y_{i}\right] .
$$

Now the $g^{\prime}$ 's are of class $C^{\mu-1}$ by Lemma 2 . Hence the $Y$ 's and, therefore, the $A$ 's, are of class $C^{\mu-2}$ by (1). By (7), (1), and (4), 
we have $A_{i}=Y_{i}=\sum_{j+1}^{2 k} X_{j} \delta_{j i}=X_{i}, \quad(i=1,2, \cdots, 2 k-1)$, at the origin. Hence $(\beta)$ follows at once from $(B)$.

It remains to show that the $A$ 's as defined above satisfy (C). It follows from (3) that

$$
\left|b_{i j}\right|=\left|a_{i j}\right| \cdot\left(\frac{\partial\left(g_{1}, \cdots, g_{2 k}\right)}{\partial\left(y_{1}, \cdots, y_{2 k}\right)}\right)^{2} \neq 0
$$

Hence we have a unique set of functions $\xi_{1}, \cdots, \xi_{2 k}$ satisfying the equations

$$
\begin{array}{ll}
\sum_{j=1}^{2 k-1} b_{i j} \xi_{j}+b_{i, 2 k} \xi_{2 k}=0, & (i=1,2, \cdots, 2 k-1), \\
\sum_{j=1}^{2 k-1}-b_{j, 2 k} \xi_{j}+0=-e^{y_{2 k}}, & \left(\text { since } b_{2 k, j}=-b_{j, 2 k}\right) .
\end{array}
$$

Now

$$
b_{j, 2 k}=\frac{\partial Y_{j}}{\partial y_{2 k}}-\frac{\partial Y_{2 k}}{\partial y_{j}}=Y_{j}=e^{y_{2 k}} A_{j}, \quad(j=1,2, \cdots, 2 k-1),
$$

and

$$
b_{i j}=e^{y_{2 k}}\left(\frac{\partial A_{i}}{\partial y_{j}}-\frac{\partial A_{j}}{\partial y_{i}}\right)=e^{y_{2 k}} c_{i j}, \quad(i, j=1,2, \cdots, 2 k-1),
$$

on account of (6) and (7). After dividing through by $e^{y_{2 k}}$, our system of $2 k$ linear equations may, therefore, be written in the form,

$$
\sum_{j=1}^{2 k-1} c_{i j} \xi_{j}+A_{i} \xi_{2 k}=0, \quad \quad \sum_{j=1}^{2 k-1} A_{j} \xi_{j}=1 .
$$

The coefficients now are independent of $y_{2 k}$. Furthermore $\xi_{2 k}=0$, as is easy to see from Cramer's rule, since the odd order determinant $\left|c_{i j}\right|$ is skew-symmetric and therefore identically zero. Hence condition $(\gamma)$ is fulfilled.

Lemma 4. Consider a Pfaffian $\omega=\sum_{i=1}^{2 k-1} X_{i} d x_{i}$ satisfying the following conditions:

(A) The $X^{\prime}$ s are of class $C^{\mu}$.

(B) None of the $X^{\prime}$ 's vanish at the origin. 
(C) The matrix $\left(a_{i j}\right)$ is such that there exists a system of functions, $\xi_{i}\left(x_{1}, \cdots, x_{2 k-1}\right)$, which satisfy the linear equations

$$
\sum_{j=1}^{2 k-1} a_{i j} \xi_{j}=0, \quad \sum_{j=1}^{2 k-1} X_{j} \xi_{j}=1 .
$$

Then there exists a non-singular transformation $x_{i}=g_{i}\left(y_{1}, \cdots\right.$, $y_{2 k-1}$ ) of the neighborhood of the origin into itself such that $\omega$ appears in terms of the $y^{\prime}$ ' in the form $\sum_{i=1}^{2 k-2} Y_{i} d y_{i}+d y_{2 k-1}$, where the $Y_{i}$ are functions of $y_{1}, \cdots, y_{2 k-2}$ (independent of $y_{2 k-1}$ ) satisfying the following conditions:

( $\alpha$ ) The $Y^{\prime}$ 's are of class $C^{\mu-2}$.

$(\beta)$ None of the Y's vanish at the origin.

Proof. Consider the equations

$$
\frac{d x_{i}}{d y_{2 k-1}}=\xi_{i}\left(x_{1}, \cdots, x_{2 k-1}\right), \quad(i=1,2, \cdots, 2 k-1) .
$$

It is clear that at least one of the $\xi$ 's does not vanish at the origin on account of $(\mathrm{C})$. Without loss of generality we assume that $\xi_{2 k-1}(0, \cdots, 0) \neq 0$. It is also clear that the $\xi$ 's may be taken to be of class $C^{\mu-1}$. We can apply Lemma 2 to (8), taking $f_{i}=\xi_{i}, n=2 k-1, \nu=\mu-1$. We show that the $g_{i}$, as there defined, satisfy the conditions of the present lemma.

For, on account of (8) and (C), we have

$$
\sum_{j=1}^{2 k-1} X_{j}\left(\frac{\partial g_{j}}{\partial y_{2 k-1}}\right)=1, \quad \sum_{j=1}^{2 k-1} a_{i j}\left(\frac{\partial g_{j}}{\partial y_{2 k-1}}\right)=0 .
$$

Hence, referring back to (1), we have $Y_{2 k-1} \equiv 1$, while (2) yields the result that

$$
\begin{aligned}
\frac{\partial Y_{i}}{\partial y_{2 k-1}} & =\frac{\partial Y_{i}}{\partial y_{2 k-1}}-\frac{\partial Y_{2 k-1}}{\partial y_{i}}=\sum_{j, l=1}^{2 k-1} a_{j l} \frac{\partial g_{j}}{\partial y_{i}} \frac{\partial g_{l}}{\partial y_{2 k-1}} \\
& =\sum_{j=1}^{2 k-1}\left[\sum_{l=1}^{2 k-1} a_{j l}\left(\frac{\partial g_{l}}{\partial y_{2 k-1}}\right)\right]\left(\frac{\partial g_{j}}{\partial y_{i}}\right)=0 .
\end{aligned}
$$

This shows that the $Y$ 's are all independent of $y_{2 k-1}$. It remains to prove that conditions $(\alpha)$ and $(\beta)$ are satisfied.

Condition $(\alpha)$ follows from (1) and the fact that, by Lemma 
2 , the $g^{\prime}$ 's are of class $C^{\mu-1}$. Condition $(\beta)$ follows from the fact that, by (1) and (4), $\left.X_{i}\right|_{0}=\left.Y_{i}\right|_{0},(i=1,2, \cdots, 2 k-2)$.

We are now ready to prove our main result.

Theorem. Consider a Pfaffian $\omega=\sum_{i=1}^{2 m} X_{i} d x_{i}$ satisfying the following conditions:

(I) The X's are of class $C^{4 m+p},(p \geqq 0)$.

(II) None of the $X$ 's vanish at the origin.

(III) The determinant $\left|a_{i j}\right|$ does not vanish at the origin.

Then there exists a non-singular transformation of coordinates, valid in the vicinity of the origin, which it leaves fixed, such that $\omega$ appears in the form $\sum_{i=1}^{m} y_{2 i} d y_{2 i-1}+d\left(\sum_{i=1}^{m} y_{2 i-1}\right)$. This transformation is of class $C^{p+1}$.

Proof. Applying Lemma 3, with $k=m$ and $\mu=4 m+p$, we write $\omega$ in the form $e^{x_{2 m}}\left(\sum_{i=1}^{2 m-1} X_{i} d x_{i}\right)$, where the new $X$ 's are independent of $x_{2 m}$ and in fact satisfy all the hypotheses of Lemma 4 , with $k=m$ and $\mu=4 m-2+p$. Hence, applying Lemma 4 , we can further simplify $\omega$, which now appears in the form

$$
e^{x_{2 m}}\left(\sum_{i=1}^{2 m-2} X_{i} d x_{i}\right)+e^{x_{2 m}} d x_{2 m-1}
$$

The new $X$ 's, by Lemma 4, satisfy all the hypotheses of Lemma 3 (with $k=m-1, \mu=4(m-1)+p$ ), except that we must verify that the new determinant $\left|a_{i j}\right|$ does not vanish. This, however, can easily be done with the help of (3) on account of the fact that the transformation in question is known to be non-singular. Hence we can successively apply Lemmas 3 and 4 again and as a result we have $\omega$ in the form

$$
e^{x_{2 m}} e^{x_{2 m-2}}\left(\sum_{i=1}^{2 m-4} X_{i} d x_{i}\right)+e^{x_{2 m}} e^{x_{2 m-2}} d x_{2 m-3}+e^{x_{2 m}} d x_{2 m-1} .
$$

The new $X$ 's are independent of $x_{2 m-3}, \cdots, x_{2 m}$ and are of class $C^{4(m-2)+p}$. This process may obviously be continued until at the end of the $m$ th application of Lemmas 3 and 4 we get $\omega$ in the form

$$
e^{x_{2} e^{x_{4}}} \cdots e^{x_{2 m}} d x_{1}+\cdots+e^{x_{2 m-2} e^{x_{2 m}}} d x_{2 m-3}+e^{x_{2 m}} d x_{2 m-1} .
$$

The transformation at the last application of Lemma 4 is of 
class $C^{p+1}$. The transformations at the previous steps are of higher class. Hence the compound transformation is of class $C^{p+1}$. Finally we make the additional transformation:

$$
\begin{aligned}
& y_{1}=x_{1}, \quad y_{2}=e^{x_{2} e^{x_{4}}} \cdots e^{x_{2 m}}-1, \\
& y_{3}=x_{3}, \quad y_{4}=e^{x_{4}} e^{x_{6}} \cdots e^{x_{2 m}}-1 \text {, } \\
& y_{2 m-1}=x_{2 m-1}, \quad y_{2 m}=e^{x_{2 m}}-1 .
\end{aligned}
$$

This transformation, which obviously is analytic with jacobian equal to 1 at the origin, leaves the origin invariant and transforms $\omega$ into the form $\left(y_{2}+1\right) d y_{1}+\cdots+\left(y_{2 m-2}+1\right) d y_{2 m-3}$ $+\left(y_{2 m}+1\right) d y_{2 m-1}$. This completes the proof.

It is scarcely necessary to add that, if (I) be replaced by the hypothesis that the $X$ 's be analytic, the transformation $T$ furnishing the desired reduction of $\omega$ will also be analytic. To see this it is only necessary to make slight obvious changes in the previous work, beginning with Lemma 2, where we take the $f$ 's and consequently the $g$ 's as analytic.

In the application to the reduction of the Pfaffian equations of dynamics to Hamiltonian form, the condition (II) is of no disadvantage. For the Pfaffian differential equations are unaltered if to each $X_{i}$ a constant is added. Assuming then that (II) holds (for each value of $t$ ) the equations may be written in variational form as follows:

$$
\delta \int\left[\sum_{i=1}^{m} y_{2 i}\left(\frac{d y_{2 i-1}}{d t}\right)+H\right] d t=0,
$$

where now, since the $X$ 's (and consequently $T$ ) depend in general periodically upon $t, H$ is not necessarily equal to $Q$, but rather, of course,

$$
H=Q+\sum_{i=1}^{2 m} X_{i}\left(\frac{d x_{i}}{d t}\right)
$$

Princeton University 